\title{
Los ruidos respiratorios en el síndrome de condensación pulmonar. Controversia en la literatura
}

\author{
Carlos David Pérez-Malagón \\ Universidad Autónoma de Aguascalientes, Aguascalientes, Ags., México
}

\section{Palabras clave \\ Ruidos respiratorios · Condensación · Síndrome}

A lo largo de mi estancia en la carrera de medicina, al estudiar el síndrome de condensación pulmonar he observado que existe controversia en la descripción de los ruidos respiratorios entre las diferentes publicaciones de literatura médica mexicana, y la literatura mundial, lo que nos produce mucha confusión entre los alumnos, residentes e incluso médicos especialistas. Para abordar este punto es importante señalar que los sonidos o ruidos respiratorios se producen por las turbulencias de aire que se forman al chocar con las variaciones anatómicas de las vías respiratorias (laringe, carinas de bronquios, bronquiolos y sacos alveolares) durante la inspiración y espiración $[1,2,3]$.

El ruido respiratorio que escuchamos al auscultar a una persona está formado a su vez por la suma de 2 ruidos. El primero es consecuencia del remolino que se forma en el espacio glótico y que se conoce como «ruido bronquial» o «glótico», ruido que es percibido solamente en ciertas regiones como son, la región traqueal, la laríngea, la esternal y a lo largo de la columna vertebral torácica, cabe aclarar, que este ruido normalmente no se percibe en la periferia del tórax durante la auscultación indirecta. El otro sonido es conocido como «ruido o murmullo vesicular», que se produce por el tránsito del aire en las vías aéreas distales, es decir, es un sonido alveolar y se logra escuchar tanto en la inspiración como en la espiración [3].

La intensidad de los ruidos respiratorios puede disminuir cuando se obstruyen las vías aéreas por líquidos o sólidos, en el enfisema, en la movilidad diafragmática disminuida o cuando existe mayor separación a la normal entre en el parénquima pulmonar y la pa- red torácica como sucede en el neumotórax, en el derrame pleural o al haber engrosamiento de la pleura $[1,2,3]$.

En el caso de la neumonía, la acumulación de líquido (exudado inflamatorio) en las vías aéreas distales produce solidificación del parénquima afectado; este cambio hace el que parénquima sea un mejor medio conductor acústico que no logra atenuar de manera normal el ruido traqueal en la periferia del tórax. La transmisión de este ruido a la periferia del tórax (zona afectada) produce un ruido grave conocido como soplo tubárico (típico de la neumonía) que impide escuchar el terso y suave murmullo vesicular $[2,4]$. $\mathrm{Al}$ profundizar en este aspecto, se han descrito tres fases anatomo-clínicas de la neumonía que se correlacionan con la auscultación. En la etapa inicial donde hay inundación de líquido (exudado) dentro de los sacos alveolares se pueden percibir estertores subcrepitantes, sin cambios en el ruido respiratorio. La siguiente fase tiene los signos clásicos de la consolidación pulmonar, en donde el ruido respiratorio per se se encuentra disminuido y se ausculta el soplo tubario. En tanto que en la tercera fase se detectan estertores inspiratorios, pero sin ruidos respiratorios [5]. Finalmente deseo concluir que en el síndrome clínico de condensación pulmonar el ruido respiratorio se encuentra disminuido como se establece en la literatura mundial [6-10]. Por alguna razón que desconozco, en la mayor parte de la literatura de autores mexicanos este concepto es opuesto [11-15]. Considero que esto deberá ser evaluado, ya que los alumnos y residentes estamos en periodo de aprendizaje y nos crea una gran confusión al leer las diferentes posturas con respecto a los ruidos respiratorios en el síndrome de condensación pulmonar lo que repercute en el correcto diagnóstico clínico de un paciente con neumonía. 


\section{Referencias}

1 Cruz Mena E, Moreno Bolton R: Aparato Respiratorio Fisiología y Clínica, ed 5, Santiago: Escuela de Medicina de Universidad Católica, 2007.

2 Grippi MA, Senior RM, Callen JP. Approach to the patient with respiratory symptoms; en Grippi MA, Elias JA, Fishman JA, et al. (eds): Fishman's Pulmonary Diseases and Disorders, ed 5, New York, NY: McGraw-Hill Education, 2015, https://accessmedicine.mhmedical.com/ content.aspx?bookid=1344\&section $\mathrm{id}=81186194$ (ultimo acceso 05.11.2021).

3 Suros Forns J, Surós Batllo A: Semiología y Propedéutica, ed 8, Barcelona: Elsevier-Masson, 2001.

4 Marrie TJ: Acute Bronchitis and CommunityAcquired Pneumonia; en Grippi MA, Elias JA, Fishman JA, et al. (eds): Fishman's Pulmonary Diseases and Disorders, ed 5, New York: Mc Graw Hill Education, New York: 2015, https:// accessmedicine.mhmedical.com/content.aspx? bookid $=1344$ \&sectionid $=81199286 \quad$ (ultimo acceso 05.11.2021).
5 Blinkhorn Jr RJ: Community-Acquired Pneumonia; en Crapo D, Glassroth J, Karlinsky JB, et al.: Baum's Textbook of Pulmonary Diseases, ed 7, Philadelphia: Lippincott Williams \& Wilkins, 2003, pp 293-316.

6 Seidel HM, Ball JW, Dains JE, et al.: Tórax y pulmones. Manual Mosby de Exploración Física, ed 7, Barcelona: Elsevier España, 2011.

7 Luna CM, Jolly EC; Aparato respiratorio; en Argente HA, Álvarez ME 8eds): Semiología médica. Fisiopatología, semiotécnica y propedéutica, ed 2, Buenos Aires: Médica Panamericana, 2005, pp 585-589.

8 Jadavji T, Law B, Lebel MH, et al.: A practical guide for the diagnosis and treatment of pediatric pneumonia. CMAJ. 1997;56:S703-11.

9 Bilkis MD, Gorgal N, Carbone M, et al.: Validation and development of a clinical prediction rule in clinically suspected communityacquired pneumonia. Pediatr Emerg Care. 2010;26:399-405.
10 Lozano JM, Steinhoff M, Ruiz JG, et al.: Clinical predictors of acute radiological pneumonia and hypoxaemia at high altitude. Arch Dis Child. 1994;71:323-327.

11 Herrera-García JC, Sánchez-Pérez R: Síndromes pleuropulmonares: de la tisiología a la neumología. Med Int Méx. 2015;31:289-295.

12 Báez-Saldaña R, Monraz-Pérez S, Fortoul-Van der Goes T, et al.: Exploración física toracopulmonar Proyecto tutorial-interactivo. Neumol Cir Tórax. 2016;75:237-252.

13 Báez R, Monraz S, Castillo P, et al.: La exploración del tórax: una guía para descifrar sus mensajes. Rev Fac Med UNAM. 2016;59: 43-57.

14 Cosio Villegas I: Síndrome de condensación pulmonar; en Cosio I, Celis A, Cosio M, et al. (eds): Aparato respiratorio, ed 17, México: Méndez, 2010, pp 215-218.

15 Villarreal-Velarde H, Cano Valle F: Signos y síntomas respiratorios; en Cano-Valle F (ed): Enfermedades del aparato respiratorio, ed 3, México: Méndez, 2013, pp 51-66. 